\title{
Feasibility of resection and plication "RAP" technique for management of medically refractory GERD in patients with altered gastric anatomy
}

(1) $\odot \ominus$

\author{
Authors \\ Patrick R. Walsh ${ }^{1,2}$, Mehul Lamba ${ }^{2}$, Petros Benias ${ }^{3}$, Abdulnasser Lafta ${ }^{2}$, George Hopkins ${ }^{1,2}$
}

Institutions

1 St. Vincent's Private Hospital Northside, Chermside, Australia

2 Royal Brisbane \& Women's Hospital, Butterfield Street, Herston, Australia

3 Division of Gastroenterology, North Shore-Long Island Jewish Medical Center, Zucker school of Medicine at Hofstra/Northwell, Northwell Health System, Manhasset, New York, United States

submitted 7.3.2021

accepted after revision 16.6.2021

Bibliography

Endosc Int Open 2021; 09: E1549-E1555

DOI 10.1055/a-1535-1279

ISSN 2364-3722

(c) 2021. The Author(s).

This is an open access article published by Thieme under the terms of the Creative Commons Attribution-NonDerivative-NonCommercial License, permitting copying and reproduction so long as the original work is given appropriate credit. Contents may not be used for commercial purposes, or adapted, remixed, transformed or built upon. (https://creativecommons.org/licenses/by-nc-nd/4.0/)

Georg Thieme Verlag KG, Rüdigerstraße 14,

70469 Stuttgart, Germany

Corresponding author

Dr. Patrick R Walsh, St. Vincent's Private Hospital Northside,

627 Rode Road, Chermside, QLD, 4032, Australia

Fax: +61738614897

pwalsh@ddq.net.au

\section{ABSTRACT}

Background and study aims Gastroesophageal reflux disease (GERD) is common, especially in patients after gas- tric surgery. Medical management of GERD is ineffective in up to $30 \%$ patients and revisional gastric surgery for management of GERD is associated with higher morbidity. We aimed to assess the safety, feasibility, and efficacy of a novel endoscopic resection and plication (RAP) anti-reflux procedure for management of medically refractory GERD in patients with altered gastric anatomy.

Patients and methods The RAP procedure involves endoscopic mucosal resection and full-thickness plication over the right posterior-medial axis extending $15 \mathrm{~mm}$ above and 20 to $30 \mathrm{~mm}$ below the squamocolumnar junction. Adverse events, technical feasibility, GERD health-related quality-of-life (GERD-HRQL) scores, and medication use were prospectively recorded.

Results Twenty consecutive patients with previous gastric surgery underwent RAP between September 2018 and August 2020 with a median follow-up of 5.7 months. The median procedure duration was 66 minutes (IQR 53.8-89.5). RAP was technically successful in 19 patients. One patient developed gastric hemorrhage from suture dehiscence, which was managed endoscopically, and four patients developed esophageal stricture requiring endoscopic dilation. Following the RAP procedure, significant improvement in GERD-HRQL score was observed (mean 26.9, $95 \% \mathrm{Cl}$ $23.36-30.55, P<0.01)$. Fourteen of 19 patients reported $>50 \%$ improvement in GERD-HRQL scores. Sixteen of 18 patients reported reduction in requirement for or cessation of antacid therapy.

Conclusions Patients with refractory GERD after gastric surgery have limited therapeutic options. We have demonstrated that the RAP procedure is feasible, safe, and clinically effective at short-term follow-up. It provides a potential alternative to revisional surgery in patients with altered gastric anatomy.

\section{Introduction}

Gastroesophageal reflux disease (GERD) is common, with a reported prevalence of up to $10 \%$ to $20 \%$ in the western world [1]. GERD results from a reduction in the lower esophageal sphincter (LES) pressure, either secondary to LES dysfunction, a crural diaphragmatic defect, or both. Moreover, GERD is a common complication following certain gastric surgeries including sleeve gastrectomy, with up to $42 \%$ of patients reporting worsening or new-onset GERD [2-4]. 
Lifestyle modification and proton pump inhibitors (PPI) are ineffective in controlling symptoms in up to $30 \%$ of patients [5]. In patients with medically refractory GERD, laparoscopic fundoplication is regarded as the gold-standard treatment. However, in patients after previous gastric surgery, owing to altered anatomy, fundoplication is often not feasible, and consequently, surgical management options are significantly limited. Therefore, an endoscopic approach seems inherently logical for this group of patients.

Various endoscopic approaches have been developed over the last two decades for use in patients with normal gastric anatomy. This includes performing endoscopic fundoplication by using proprietary devices such as transoral incisionless fundoplication [6-8], GERDx [9] and MUSE [10-12] which have shown improved symptom control and a reduced requirement for use of PPI in the short to medium term. Other endoscopic therapies, including radiofrequency wave therapy at gastroesophageal junction (GEJ), have been shown to be beneficial in the short term [13-17]. Inoue et al described anti-reflux mucosectomy in 10 patients with medically refractory GERD with normal gastric anatomy, resulting in excellent symptom improvement and 24-hour $\mathrm{pH}$ study at 2 months [3].

Benias et al described a new technique, resection and plication (RAP), involving a combination of endoscopic mucosal resection (EMR) and full-thickness plication at the left posterolateral axis of the GEJ to strengthen the LES [18]. Because the RAP technique does not involve plication of the fundus, this procedure can be offered in patients with altered gastric anatomy. More recently, Benias et al has described modifications of the original RAP procedure, by performing mucosectomy and plication at the right posterior-medial axis of the GE] [19].

In this study, we aimed to assess the safety, feasibility, and short-term clinical efficacy of the RAP procedure for management of medically refractory GERD in patients after previous gastric surgery.

\section{Patients and methods}

\section{Patient selection}

Consecutive patients with medically refractory GERD referred for consideration of RAP procedure between September 2018 and August 2020 were included. Patients were defined as having medically refractory GERD if they continued to experience symptoms despite maximally tolerated PPI therapy. All patients had previously been seen by the surgical team and surgery was offered for management of reflux. RAP was offered if patients were older than age 18 years and able to provide written informed consent. Contraindications to the RAP procedure included a hiatal hernia $>3 \mathrm{~cm}$, gastric tube stenosis in patients with prior laparoscopic sleeve gastrectomy (LSG), and anticoagulation therapy that could not be interrupted.

\section{Pre-procedural work-up}

All patients underwent a baseline endoscopy for assessment of hiatal hernia and exclusion of gastric body stenosis in those who were post-LSG. The size of the hiatal hernia was measured from the diaphragmatic pinch to the GEJ (the proximal margin of the gastric folds). In patients post-LSG in whom significant gastric stenosis could not be excluded at baseline endoscopy, additional investigations were undertaken at the discretion of the endoscopist, including barium swallow and/or endoscopic functional luminal imaging probe (EndoFLIP; Crospon Ltd, Galway, Ireland). A GERD health related quality-of-life questionnaire (GERD-HRQL) [20] was completed at baseline.

\section{Procedure}

All procedures were performed by one endoscopist (PW). The procedure was performed under general anesthesia with broad-spectrum antibiotic cover (ceftriaxone $2 \mathrm{~g}$ intravenous or equivalent) in a hospital-based day procedure endoscopy unit. Analgesia and antiemetics were given routinely prior to extubation.

Using a gastroscope (Olympus GIF-1TH190), a trapezoidal or pyramidal shaped mucosectomy (narrow proximally and widening distally) was performed using a multiband mucosal resection device (Duette; Cook Medical, Limerick, Ireland) and a standard submucosal lift technique using chromo-gelofusineadrenaline solution $(2 \mathrm{ml}$ methylene blue and $2 \mathrm{~mL}$ of 1 in 1,000 adrenaline in $500 \mathrm{~mL}$ gelofusine). The mucosectomy was commenced at approximately $15 \mathrm{~mm}$ proximal to the squamocolumnar junction along the right posteromedial axis, extending 20 to $30 \mathrm{~mm}$ distally on gastric side along the lesser curvature. The mucosectomy was narrow proximally, widening as it extended distally but staying less than $50 \%$ of the circumference ( $\triangleright$ Fig. 1 and $\triangleright$ Fig. 2 ). When compared to the gastric cardia, the mucosectomy over GEJ was narrower to reduce the risk of esophageal stricturing. To control intraprocedural bleeding, monopolar haemostatic forceps (Coagrasper; Olympus medical systems) were used with electrocautery generator settings: soft coagulation, 80 watts, effect 4 (ERBE; Erlangen, Germany).

The Apollo overstitch device (Apollo Endosurgery; Austin, Texas) was mounted onto a double-channel therapeutic endoscope (Olympus GIF-2TH180). Suturing was performed in forward view and a single suture (2-0 standard Apollo prolene suture) was directed through the exposed muscle fibers in a fullthickness manner as a running suture, in a predetermined pattern ( $\triangleright$ Fig. 1). The suture was started distally at the anteriormedial aspect of the EMR defect in the 1 to 2 o'clock position with the needle passed through mucosa and exiting into the EMR defect. A combination of scope rotation and suction was used to facilitate deep bites, and the tissue grasping device (Tissue Helix; Apollo Endosurgery, Austin, Texas, United States) was used if required.

The suture was then taken to the distal posterior corner of the EMR defect ( 6 to 7 o'clock position) and the needle passed from the EMR defect out through normal mucosa using the same endoscopic maneuvers. Following this the suture was taken back to the anterior aspect of the EMR defect working proximally and similar process followed until between four and six bites had been performed. Care was taken not to cross the suture, the bites were opposite each other, and the final bite was through the most proximal part of the EMR defect. Finally, the needle was dropped, and adequate tension was applied to the suture. Once satisfactory defect apposition and plication were 


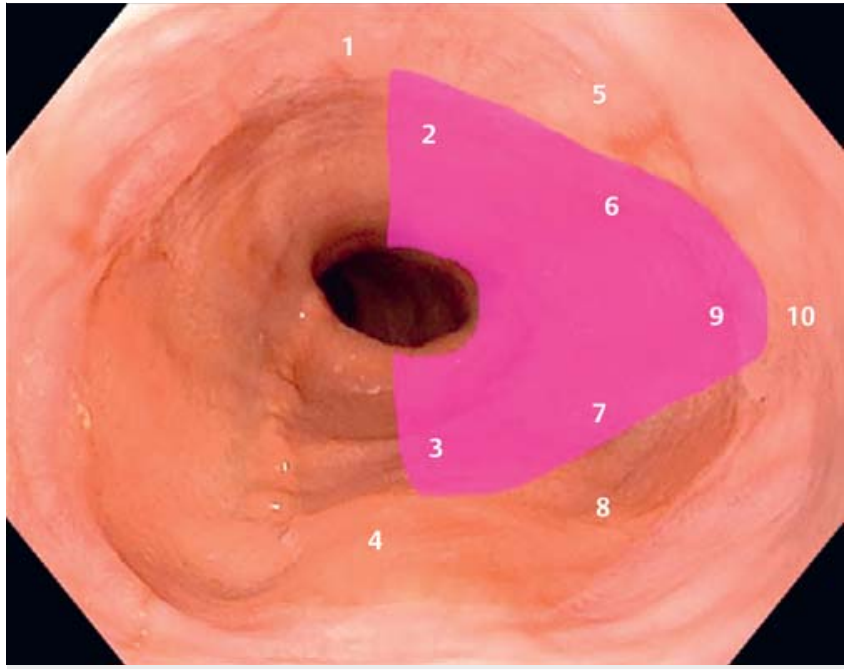

Fig. 1 Conceptual diagram of modified RAP procedure. The shaded area indicates the site for endoscopic mucosal resection. The numbers represent the order in which mucosal bites are taken to accomplish full-thickness plication.

achieved, a cinch was deployed. If the EMR defect could not be closed with one suture (for example, due to technical issues preventing a minimum of four bites being taken) a second suture was placed.

\section{Post-procedure care}

Patients were either admitted overnight for observation or discharged on the same day. Patients were advised to stay on thinliquid diet for 2 weeks, pureed diet for 2 weeks, followed by a soft diet for 2 weeks, before progressing to normal diet. Patients were asked to continue their pre-procedure PPI dose for 4 weeks, then halve the dose for next 4 weeks and subsequently wean off it completely based on symptom control. Repeat endoscopy was not routinely performed in all patients. We reported adverse events (AE) as either intraprocedural, within 2 weeks or greater than 30 days after the endoscopic procedure, as described in the American society of Gastrointestinal Endoscopy lexicon for reporting endoscopic AE [21].

Demographic data, medication history, body weight, and GERD-HRQL were obtained at baseline. In addition, GERDHRQL scores were recorded prospectively at routine post-procedure follow-up appointments. Data on procedure-related complications were recorded at each follow-up appointment. PPI use was recorded as omeprazole equivalents based on relative drug potency as follows: 1.0, 0.23, 0.9, 1.6 and 1.82 for omeprazole, pantoprazole, lansoprazole, esomeprazole and rabeprazole, respectively, as described by Kirchheiner et al [22].

This study was approved by the local ethics board.

\section{Results}

Twenty consecutive patients with prior gastric surgery underwent the RAP procedure between September 2018 and June 2020 , with a median clinical follow-up of 5.7 months. The me-

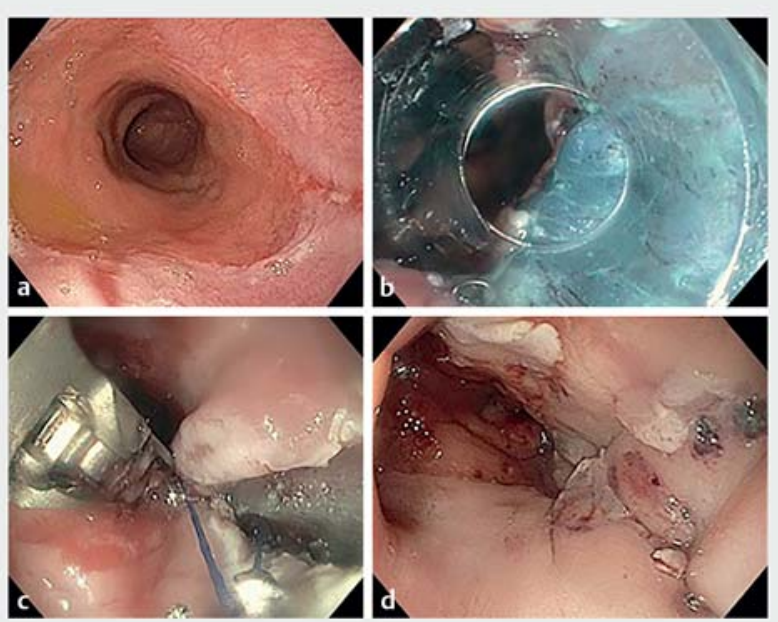

- Fig. 2 Steps in RAP procedure. a Baseline endoscopy demonstrating a small hiatal hernia and LA Grade A reflux esophagitis. b Band endoscopic mucosal resection (EMR) with Duette device over right posterior-medial axis after chromogelofusine-adrenaline lift. c Plication and closure of EMR defect using Overstitch device mounted on double-channel therapeutic gastroscope. d Completion of RAP procedure with total closure of EMR defect.

dian age was 46.5 years (IQR 37.2-64) and 18 patients (90\%) were female. Eleven patients had undergone LSG: two biliarypancreatic diversion, one single anastomosis bypass graft, four Roux-en-Y bypass (RYGB) graft, and two patients had reversal of previous RYGB ( $\triangleright$ Table 1 ). The median duration from last gastric surgery to RAP was 2.8 years (IQR 1.7-5.3).

At baseline gastroscopy, 10 patients (50\%) had evidence of active reflux esophagitis despite maximal PPI therapy. A small hiatal hernia $(<3 \mathrm{~cm})$ was noted in nine patients $(45 \%)$. In patients with previous LSG, barium swallow or EndoFLIP were performed in five and four patients, respectively, to exclude gastric body stenosis.

The median procedure duration was 66 minutes (IQR 53.8$89.5)$. The median procedure duration in the last 10 patients was shorter, compared to the first 10 patients (52 compared to 89 minutes, $P<0.001)$. RAP was technically successful in 19 patients (95\%). Technical failure was observed in one patient, where at suture deployment, complete closure of the lower esophageal lumen with axial torsion was noted. The suture was immediately cut. Repeat sutures were not undertaken in this patient.

In 14 patients a single suture was used; the other six required two sutures to close the EMR defect. Twelve patients (60\%) were discharged the same day, six (30\%) were discharged the following day. One patient each were discharged on day 2 and day 13. The last patient had developed dysphagia due to esophageal stenosis requiring inpatient gastroscopy for removal of sutures and dilation. 


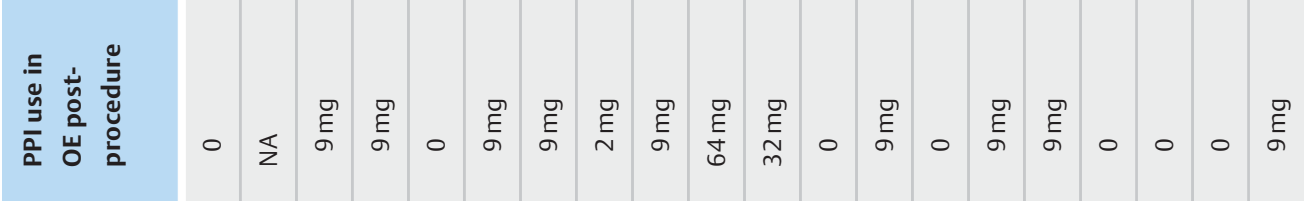

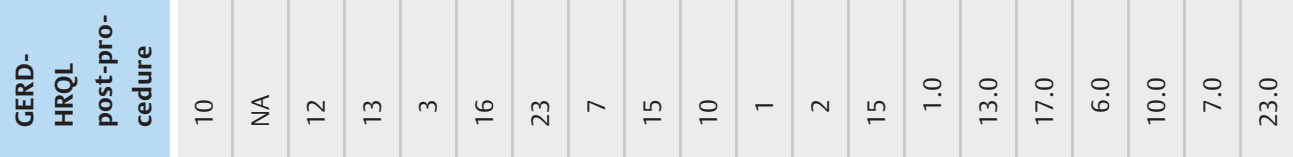

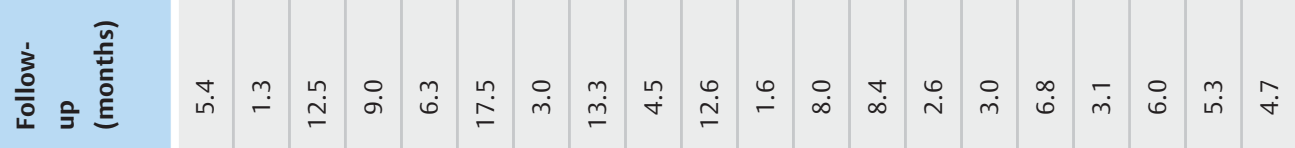

产

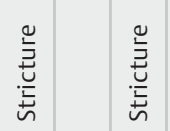

总

竞

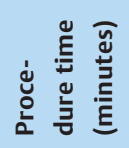

$\stackrel{\sim}{\sim}$ 【

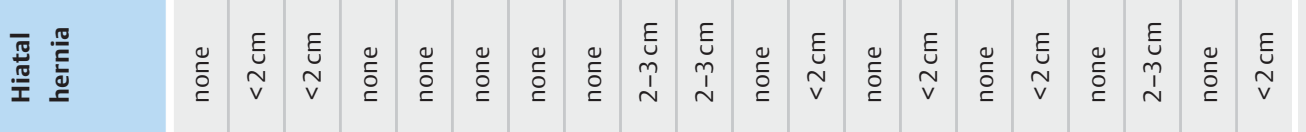

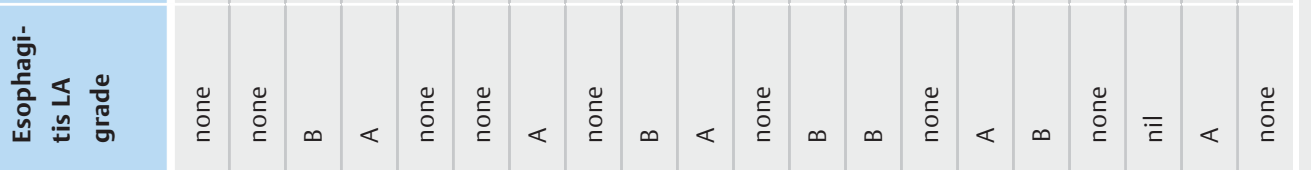

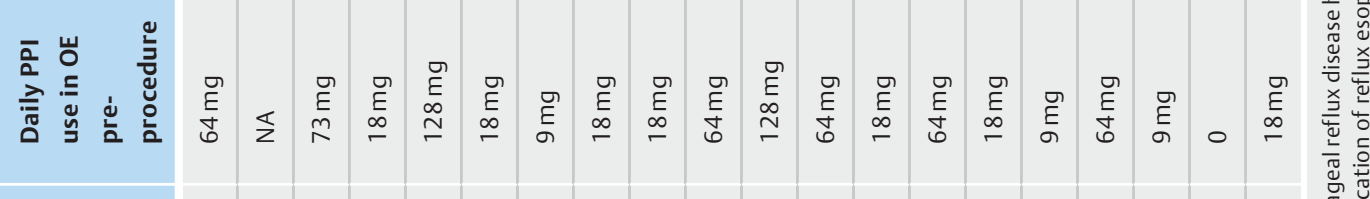

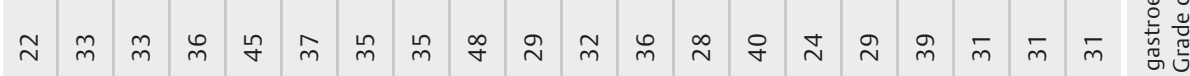

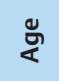

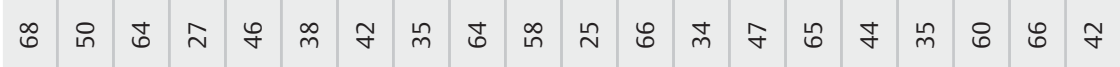

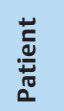

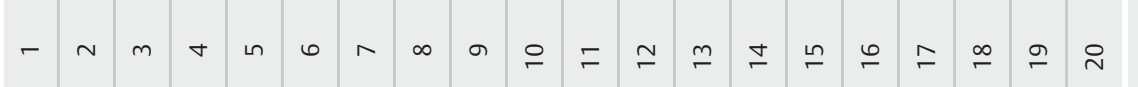




\section{Adverse events}

There were no major intraprocedural AEs. There were five AEs within 2 weeks of endoscopy, there were no delayed AEs reported.

One patient presented with large-volume hematemesis after an episode of forceful vomiting 5 days post-procedure. The patient was hemodynamically stable at presentation. Urgent gastroscopy showed gastric hemorrhage caused by suture dehiscence. Endoscopic homeostasis was achieved using monopolar haemostatic forceps (Coagrasper; Olympus Medical Systems). The following day a repeat plication was undertaken with the overstitch device as described above.

Four patients (18\%) developed dysphagia requiring endoscopic intervention. In two patients, sutures were cut at 4 and 5 weeks after procedure. Dilatation was performed with a controlled radial expansion dilator (CRE; Boston Scientific Corp, USA) at a median of 5.5 weeks (range 4-9 weeks) up to 13- to 15-mm diameter. An average of two dilatation procedures were required per patient to achieve adequate symptom relief.

\section{GERD-HRQL score}

Serial GERD-HRQL scores were available for 19 of 20 patients ( $\vee$ Fig.3). One patient was lost to follow-up. The median GERD-HRQL score at baseline was 34 (IQR 30.5-36.2). Repeat GERD-HRQL scores were calculated after a median interval of 23 weeks (IQR 14.7-31.5). After RAP procedure, the mean reduction in GERD-HRQL score was 26.9 (95\% Cl 23.36-30.55), with a post-RAP median GERD-HRQL of 10 (IQR 6.5-15). In nine patients, GERD-HRQL scores were assessed again at 6-12 months after procedure, which were similar to their scores assessed within 6 months post-RAP $(P=0.68)$. Overall, 14 of 19 patients reported $>50 \%$ improvement in GERD-HRQL scores.

Eighteen patients were on PPI therapy pre-procedure, at a median daily dose (in omeprazole equivalents) of $18 \mathrm{mg}$ (IQR 18-64). After the RAP procedure, six patients reported complete cessation of PPI use, while another 10 patients reported reduction in PPI dosage after the RAP procedure. Overall, medi- an daily PPI dose (in omeprazole equivalents) post-RAP procedure was $9 \mathrm{mg}$ (IQR 0-9).

No patients required surgery for management of reflux symptoms. The remaining two patients continued to be on same dose of PPI post-procedure.

\section{Discussion}

Patients with medically refractory reflux post gastric surgery represent a challenging cohort with limited therapeutic options. Conventional surgical fundoplication, while a gold-standard treatment in patients with normal gastric anatomy, is often not possible, especially after gastric surgery involving removal of fundus such as after LSG. In many cases, conversion to RYGB is the only possible treatment for medically refractory reflux. However, revisional bariatric surgery is associated with significantly increased procedure related complications and overall morbidity [23-26]. Consequently, there is a need for a safe, effective, and minimally invasive therapy for such patients. In this study, we present our experience in performing RAP procedure in 20 consecutive patients with altered gastric anatomy presenting with medically refractory GERD.

RAP is a novel procedure, first described by Benias et al in 2018 [18]. It involves a combination of EMR and full-thickness suture to allow plication and induce controlled scarring and augmentation of the LES. In contrast to the original RAP procedure in which EMR and plication were targeted at the posteriorlateral axis, we used the modified RAP technique [19], in which EMR and plication were performed at the posterior-medial axis. Because the clasp and sling fibers in the stomach meet at the posterior-medial region of the gastric cardia, we hypothesize that scarring and plication in this area result in higher pressure at the LES. How each technique of RAP influences the pressure dynamics at LES remains to be objectively assessed.

In our cohort, the RAP procedure was clinically effective in all 19 patients for whom both pre-procedure and post-procedure GERD-HRQL scores were available. The GERD-HRQL score improved in all patients, with an average score reduction of

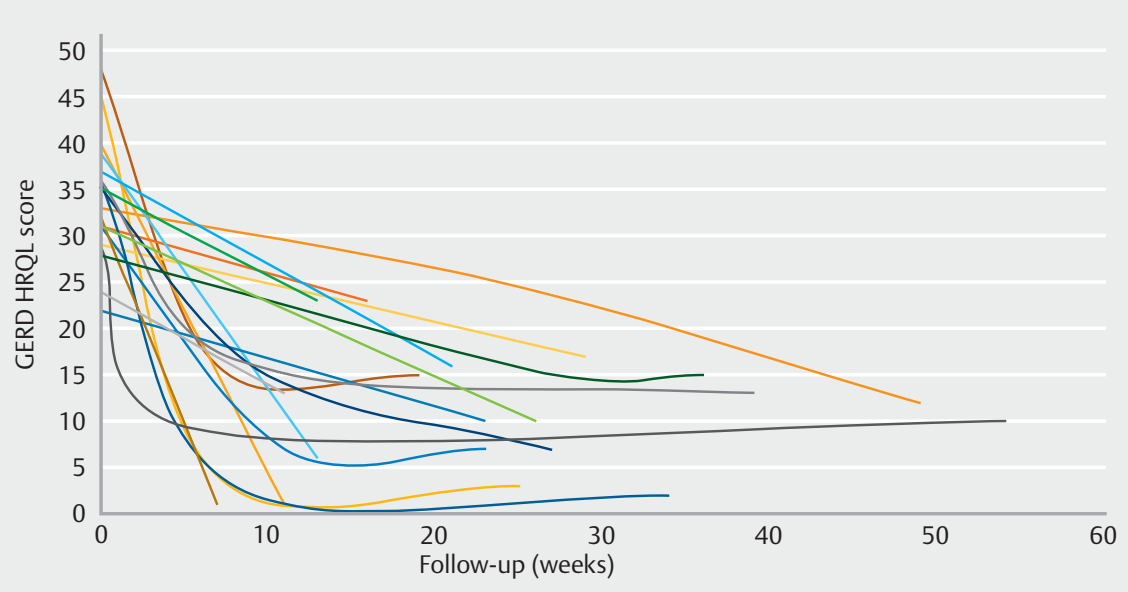

- Fig. 3 Change in GERD-HRQL Score. 
26.9 (95\% Cl 23.36-30.55). The improvement in quality-of-life score was reflected in reduced requirement for PPI therapy. Eighteen patients reported regular PPI usage prior to the procedure. Of these, six reported complete cessation of PPI use, while 10 reported a reduction in PPI dosage.

One patient developed gastric hemorrhage associated with suture dehiscence at day 5 post-procedure. Endoscopic hemostasis was successfully achieved, and repeat plication undertaken the following day. Dysphagia was observed in four patients. In two of these patients, the plication resulted in significant narrowing at the LES channel. The sutures were cut with a Loop Cutter (Olympus medical systems) in two patients. A through the scope balloon dilator was used to gradually dilate LES in four patients at a median interval of 5.5 weeks after RAP. After an average of two dilatation procedures per patient, complete resolution of dysphagia was observed, with no reoccurrence during the observation period. Dysphagia has been previously documented after ARMS and RAP technique and likely results from stricturing at LES [3, 18, 27-29]. As demonstrated in previous studies, dysphagia is manageable endoscopically in all patients and does not appear to recur after an initial adequate dilation.

LSG is the most commonly performed bariatric surgery procedure worldwide [30,31]. GERD is a well-recognized complication after LSG, resulting from division of sling fibers and damage to the phreno-esophageal ligaments, leading to impairment in LES function and reduced distensibility of gastric lumen, leading to an increase in intragastric pressure [32]. Denovo GERD is seen in up to $23 \%$ of patients and Barrett's esophagus has been noted in up to $8 \%$ patients after LSG [2]. Therefore, there is a growing unmet need to manage reflux in postLSG patients, and endoscopic treatment using RAP can potentially provide a safe and effective therapy. Moreover, because other endoscopic fundoplication procedures require retroflexed endoscope or the presence of the fundus, they may not be feasible in patients with altered gastric anatomy. The RAP procedure is performed in a forward en-face endoscopic view, and therefore, provides a distinct advantage in endoscopic management of GERD in patients with prior gastric surgery.

The main limitation of our study was that a 24-hour $\mathrm{pH}$ study and manometry were not used to assess change from baseline in LES function and quantitative assessment of reflux after the RAP procedure. This was not a funded study, therefore, undertaking additional investigations was not always possible due to associated financial cost to patients. Moreover, the main purpose of our study was to demonstrate safety and feasibility of undertaking RAP in patients with previous gastric surgery and assess of improvement in reflux-related symptoms. A separate study assessing 24-hour $\mathrm{pH}$ and manometry pre-procedure and post-RAP is needed to assess change in LES pressure dynamics. Second, the follow-up period in our study was relatively short, because RAP is a relatively new procedure and long-term follow-up data are not available. It remains unknown whether improvement in reflux related symptoms will be sustained in the long term.

\section{Conclusions}

In this prospective, single-center study, we reported our initial experience with using the novel RAP procedure to manage medically refractory reflux disease in patients with prior gastric surgery. We demonstrated that the RAP procedure is feasible, safe, improves GERD-related QoL, and reduces PPI usage in the short term. It provides an interesting therapeutic option for management of refractory GERD after gastric surgery, without precluding the possibility of potential revisional surgery if needed.

\section{Competing interests}

Dr Patrick R Walsh and Dr P C Benias are both consultants for Apollo Endosurgery.

\section{References}

[1] Dent J, El-Serag HB, Wallander MA et al. Epidemiology of gastro-oesophageal reflux disease: a systematic review. Gut 2005; 54: 710-717

[2] Yeung KTD, Penney N, Ashrafian L et al. Does sleeve gastrectomy expose the distal esophagus to severe reflux? a systematic review and meta-analysis Ann Surg 2020; 271: 257-265

[3] Inoue $\mathrm{H}$, Ito $\mathrm{H}$, Ikeda $\mathrm{H}$ et al. Anti-reflux mucosectomy for gastroesophageal reflux disease in the absence of hiatus hernia: a pilot study. Ann Gastroenterol 2014; 27: 346-351

[4] Alvarenga ES, Lo Menzo E, Szomstein S et al. Safety and efficacy of 1020 consecutive laparoscopic sleeve gastrectomies performed as a primary treatment modality for morbid obesity. A single-center experience from the metabolic and bariatric surgical accreditation quality and improvement program. Surg Endosc 2016; 30: $2673-$ 2678

[5] Scarpellini E, Ang D, Pauwels A et al. Management of refractory typical GERD symptoms. Nat Rev Gastroenterol Hepatol 2016; 13: 281294

[6] McCarty TR, Itidiare M, Njei B et al. Efficacy of transoral incisionless fundoplication for refractory gastroesophageal reflux disease: a systematic review and meta-analysis. Endoscopy 2018; 50: 708-725

[7] Hunter JG, Kahrilas PJ, Bell RC et al. Efficacy of transoral fundoplication vs omeprazole for treatment of regurgitation in a randomized controlled trial. Gastroenterology 2015; 148: 324-33 e5

[8] Hakansson B, Montgomery M, Cadiere GB et al. Randomised clinical trial: transoral incisionless fundoplication vs. sham intervention to control chronic GERD. Aliment Pharmacol Ther 2015; 42: 1261-1270

[9] Weitzendorfer M, Spaun GO, Antoniou SA et al. Clinical feasibility of a new full-thickness endoscopic plication device (GERDx) for patients with GERD: results of a prospective trial. Surg Endosc 2018; 32: 25412549

[10] Zacherl ], Roy-Shapira A, Bonavina L et al. Endoscopic anterior fundoplication with the Medigus Ultrasonic Surgical Endostapler (MUSE) for gastroesophageal reflux disease: 6-month results from a multi-center prospective trial. Surg Endosc 2015; 29: 220-229

[11] Kim HJ, Kwon Cl, Kessler WR et al. Long-term follow-up results of endoscopic treatment of gastroesophageal reflux disease with the MUSE endoscopic stapling device. Surg Endosc 2016; 30: 3402-3408

[12] Roy-Shapira A, Bapaye A, Date $S$ et al. Trans-oral anterior fundoplication: 5-year follow-up of pilot study. Surg Endosc 2015; 29: 37173721 
[13] Perry KA, Banerjee A, Melvin WS. Radiofrequency energy delivery to the lower esophageal sphincter reduces esophageal acid exposure and improves GERD symptoms: a systematic review and meta-analysis. Surg Laparosc Endosc Percutan Tech 2012; 22: 283-288

[14] Corley DA, Katz P, Wo JM et al. Improvement of gastroesophageal reflux symptoms after radiofrequency energy: a randomized, shamcontrolled trial. Gastroenterology 2003; 125: 668-6676

[15] Fass R, Cahn F, Scotti DJ et al. Systematic review and meta-analysis of controlled and prospective cohort efficacy studies of endoscopic radiofrequency for treatment of gastroesophageal reflux disease. Surg Endosc 2017; 31: 4865-4882

[16] Lipka S, Kumar A, Richter JE. No evidence for efficacy of radiofrequency ablation for treatment of gastroesophageal reflux disease: a systematic review and meta-analysis. Clin Gastroenterol Hepatol 2015; 13: 1058-1067 e1

[17] Triadafilopoulos G. Stretta: a valuable endoscopic treatment modality for gastroesophageal reflux disease. World J Gastroenterol 2014; 20: 7730-7738

[18] Benias PC, D'Souza L, Lan G et al. Initial experience with a novel resection and plication (RAP) method for acid reflux: a pilot study. Endosc Int Open 2018; 6: E443-E449

[19] Raphael KL, Walsh P, Benias PC. Innovations in endoscopic therapy for gastroesophageal reflux disease. Gastrointest Endosc Clin N Am 2020 30: 291-307

[20] Velanovich V, Vallance SR, Gusz JR et al. Quality of life scale for gastroesophageal reflux disease. J Am Coll Surg 1996; 183: 217-224

[21] Cotton PB, Eisen GM, Aabakken L et al. A lexicon for endoscopic adverse events: report of an ASGE workshop. Gastrointest Endosc 2010; 71: $446-454$

[22] Kirchheiner ], Glatt S, Fuhr U et al. Relative potency of proton-pump inhibitors-comparison of effects on intragastric $\mathrm{pH}$. Eur J Clin Pharmacol 2009; 65: 19-31
[23] te Riele WW, Sze YK, Wiezer M] et al. Conversion of failed laparoscopic gastric banding to gastric bypass as safe and effective as primary gastric bypass in morbidly obese patients. Surg Obes Relat Dis 2008; 4: 735-739

[24] Roller JE, Provost DA. Revision of failed gastric restrictive operations to Roux-en-Y gastric bypass: impact of multiple prior bariatric operations on outcome. Obes Surg 2006; 16: 865-869

[25] Stefanidis D, Malireddy K, Kuwada T et al. Revisional bariatric surgery: perioperative morbidity is determined by type of procedure. Surg Endosc 2013; 27: 4504-4510

[26] Khoursheed MA, Al-Bader IA, Al-asfar FS et al. Revision of failed bariatric procedures to Roux-en-Y gastric bypass (RYGB). Obes Surg 2011; 21: 1157-1160

[27] Monino L, Gonzalez JM, Vitton V et al. Antireflux mucosectomy band in treatment of refractory gastroesophageal reflux disease: a pilot study for safety, feasibility and symptom control. Endosc Int Open 2020; 8: E147-E154

[28] Debourdeau A, Vitton V, Monino L et al. Antireflux mucosectomy band (ARM-b) in treatment of refractory gastroesophageal reflux disease after bariatric surgery. Obes Surg 2020; 30: 4654-4658

[29] Patil G, Dalal A, Maydeo A. Feasibility and outcomes of anti-reflux mucosectomy for proton pump inhibitor dependent gastroesophageal reflux disease: First Indian study (with video). Dig Endosc 2020; 32: 745-752

[30] Ponce ], DeMaria EJ, Nguyen NT et al. American Society for Metabolic and Bariatric Surgery estimation of bariatric surgery procedures in 2015 and surgeon workforce in the United States. Surg Obes Relat Dis 2016; 12: 1637-1639

[31] Buchwald H, Oien DM. Metabolic/bariatric surgery worldwide 2011 Obes Surg 2013; 23: 427-436

[32] Mahawar KK, Carr WR, Jennings N et al. Simultaneous sleeve gastrectomy and hiatus hernia repair: a systematic review. Obes Surg 2015; 25: 159-166 